\section{Ausbildung der Ärzte im Bereich Strahlenschutz zum Sachverständigen}

\section{Rechtsgrundlagen}

Das Strahlenschutzgesetz (StSG vom 22. März 1991) und die Strahlenschutzverordnung (StSV vom 22. Juni 1994) verlangen in Artikel 18, dass Ärztinnen und Ärzte, die eine Bewilligung zum Betreiben einer Röntgenanlage erhalten wollen, über eine vom BAG anerkannte Ausbildung in Strahlenschutz und Röntgentechnik verfügen müssen.

Korrespondenz:

Jürg Marti

BAG, Abteilung und Radiopharmaceutika

Dienst Ausbildung Strahlenschutz

CH-3003 Bern

E-mail: juerg.marti@bag.admin.ch
Ärztinnen und Ärzte, die eine Bewilligung zum Betreiben einer Röntgenanlage nach dem 1. Oktober 1994 erhalten haben, müssen einen 5tägigen Kurs zur Erlangung des Sachverstands absolvieren. Die Voraussetzung zum Kursbesuch ist das eidgenössische Arztdiplom oder eine gleichwertige, anerkannte ausländische Ausbildung.

\section{Anerkennung der Ausbildung durch das BAG}

Diese Ausbildung, die zur Erlangung des Sachverstandes nach Artikel 18 der Strahlenschutzverordnung notwendig ist, wurde vom BAG anerkannt. Die Ausbildungsstätte stellt nach erfolgreich absolviertem Kurs ein vom BAG anerkanntes Zertifikat aus.

\section{Anmeldung/Information}

Anmeldeformulare können direkt bei den angegebenen Ausbildungsstätten bezogen werden, wo ebenfalls weitere Informationen über die Kursorganisation (Ort und Zeit), die definitive Anmeldung bzw. die Kurskosten (Richtpreis Fr. 1800.-) erhältlich sind.

\title{
Sachverständigenkurse
}

Von den untenstehenden Ausbildungsstätten werden im Jahre 2001 folgende Kurse angeboten:

\begin{tabular}{lll}
\hline Institution & Kurs & Ort \\
\hline Villigen & KW 40: 1.-5.10.2001 (ganze Woche) & Paul Scherrer Institut, \\
& & Schule für Strahlenschutz, \\
& Sekretariat: Frau H. Schmid, 5232 Villigen PSI, \\
& Tel. $0563102500(9-11.30$ h), Fax 0563104191
\end{tabular}

Zürich Nr. 2: 25.-27.10. + 1.-3.11.2001

\author{
Universität Zürich, \\ Institut für diagnostische Radiologie, \\ Herr PD Dr. C. Michel, \\ Postfach, Rämistrasse 100, 8091 Zürich, \\ Tel. 0125596 02, Fax 012559604
}




\section{Cours de formation en radioprotection et en technique radiologique destinés aux médecins non radiologues}

\begin{abstract}
Objectifs
$\mathrm{Au}$ terme de l'ordonnance du 22 juin 1994 sur la radioprotection (ORaP), il est exigé, à l'article 18, que les médecins désirant obtenir une autorisation pour l'exploitation d'une installation à rayons X à usage médical possédent, pour exercer leur fonction d'expert local de radioprotection, "une formation en radioprotection et en technique radiologique sanctionnée par un examen et reconnue par l'Office fédéral de la santé publique (OFSP)».

Pour les médecins ayant fait leur demande d'autorisation après la date de mise en vigueur de l'ordonnance ( $1^{\mathrm{er}}$ octobre 1994), une formation théorique et pratique d'une semaine est prévue.

Les cours présentés par la Fondation PRORAME correspondent aux exigences de l'OFSP et sont reconnus.
\end{abstract}

Correspondance:

Jürg Marti

OFSP, Division Radioprotection

Service formation et produits radiopharmaceutiques

CH-3003 Berne

E-mail: juerg.marti@bag.admin.ch

\section{Inscription}

Condition d'admission

Le diplôme fédéral de médecine, ou une formation étrangère équivalente et reconnue.

\section{Programmes détaillés}

Les programmes détaillés (avec formulaire d'inscription) peuvent être obtenus sur demande à l'Institut de radiophysique appliquée, Grand-Pré 1, 1007 Lausanne.

Délai d'inscription

Deux mois avant le cours; l'inscription sera confirmée.

Finance d'inscription

La finance d'inscription est de Fr. 1800.- par cours; elle couvre l'enseignement et la documentation distribuée. L'entretien (repas, hôtel) et les déplacements ne sont pas compris dans ces prestations.

\section{Lieu et dates}

Les dates et le lieu du prochain cours pour la Suisse romande sont les suivantes:

\begin{tabular}{lll}
\hline Institution & Dates & Lieux \\
\hline Fondation PRORAME & 10.-14.12.2001 & Genève \\
\hline
\end{tabular}

\section{Information}

Des informations complémentaires peuvent être obtenues à l'adresse suivante: Institut de radiophysique appliquée, Grand-Pré 1, 1007 Lausanne, tél. 021 62334 34, fax 0216233435. 